\title{
EXISTENCE THEOREMS FOR A SECOND ORDER M-POINT BOUNDARY VALUE PROBLEM AT RESONANCE
}

\author{
CHAITAN P. GUPTA
}

\author{
Department of Mathematics \\ University of Nevada, Reno \\ Reno, NV 89557
}

(Received October 15, 1993 and in revised form May 3, 1994)

\begin{abstract}
Let $f:[0,1] \times R^{2} \rightarrow R$ be a function satisfying Caratheodory's conditions and $e(t) \epsilon$ $L^{1}[0,1]$. Let $\eta \in(0,1), \xi_{i} \in(0,1), a_{i} \geq 0, i=1,2, \cdots, m-2$, with $\sum_{t=1}^{m-2} a_{i}=1,0<\xi_{1}<\xi_{2}<$ $\cdots<\xi_{m-2}<1$ be given. This paper is concerned with the problem of existence of a solution for the following boundary value problems

$x^{\prime \prime}(t)=f\left(t, x(t), x^{\prime}(t)\right)+e(t), 0<t<1$,

$x^{\prime}(0)=0, x(1)=x(\eta)$,

$x^{\prime \prime}(t)=f\left(t, x(t), x^{\prime}(t)\right)+e(t), 0<t<1$,

$x^{\prime}(0)=0, x(1)=\sum_{i=1}^{m-2} a, x\left(\xi_{i}\right)$.

Conditions for the existence of a solution for the above boundary value problems are given using Leray Schauder Continuation theorem.

Keywords and Phrases: three-point boundary value problem, m-point boundary value problem, Leray Schauder Continuation theorem, Caratheodory's conditions, Arzela-Ascoli Theorem.

AMS(MOS) Subject Classification: 34B10, 34B15, 34G20.
\end{abstract}

\section{INTRODUCTION.}

Let $f:[0,1] \times R^{2} \rightarrow R$ be a function satisfying Caratheodory's conditions, $e:[0,1] \rightarrow R$ be a function in $L^{1}[0,1], a_{i} \geq 0, \xi_{i} \in(0,1), i=1,2, \cdots, m-2$ with $\sum_{i=1}^{m-2} a_{i}=1,0<\xi_{1}<\xi_{2}<\cdots$, $<\xi_{m-2}<1$ and $\eta \in(0,1)$ be given. We study the problem of existence of solutions for the following boundary value problems

$$
\begin{gathered}
x^{\prime \prime}(t)=f\left(t, x(t), x^{\prime}(t)\right)+e(t), 0<t<1, \\
x^{\prime}(0)=0, x(1)=x(\eta), \\
x^{\prime \prime}(t)=f\left(t, x(t), x^{\prime}(t)\right)+e(t), 0<t<1, \\
x^{\prime}(0)=0, x(1)=\sum_{i=1}^{m-2} a_{i} x\left(\xi_{i}\right) .
\end{gathered}
$$

It is well-known, (see, e.g. [1]), that if $x \in C^{1}[0,1]$ satisfies the boundary conditions in (2), with the $a_{i}$ 's as above, then there exists an $\eta \in\left[\xi_{1}, \xi_{m-2}\right]$, depending on $x \in C^{1}[0,1]$, such that

$$
x(1)=x(\eta) .
$$

Accordingly, it seems that one can study the problem of existence of a solution for the boundary value problem (2) using the a priori estimates obtained for the three-point boundary value problem (1), as it was done in [2], [3], [4]. But here the m-point boundary value problem (2) happens to be at resonance in the sense that the associated linear homogeneous boundary value problem

$$
\begin{gathered}
x^{\prime \prime}(t)=0,0<t<1, \\
x^{\prime}(0)=0, x(1)=\sum_{i=1}^{m-2} a_{i} x\left(\xi_{i}\right),
\end{gathered}
$$

has $x(t)=A, A \in R$, as a non-trivial solution, since $\sum_{i=1}^{m-2} a_{i}=1$. The result is that $e(t) \in L^{1}[0,1]$ has to be such that $\sum_{i=1}^{m-2} a_{1}\left[\int_{0}^{\xi_{1}}\left(1-\xi_{i}\right) e(s) d s+\int_{\xi_{1}}^{1}(1-s) e(s) d s\right]=0$, (in view of the nonlinear Fredholm 
alternative), so even though there exists an $\eta \in\left[\xi_{1}, \xi_{m-2}\right]$ such that $\int_{0}^{1}(1-\eta) e(s) d s+\int_{\eta}^{1}(1-s) e(s) d s=$ $\sum_{t=1}^{m-2} a_{1}\left[\int_{0}^{\xi_{1}}\left(1-\xi_{1}\right) e(s) d s+\int_{\xi_{1}}^{1}(1-s) e(s) d s\right]=0$, since $\sum_{t=1}^{m-2} a_{1}=1$, this $\eta$ is not necessarily the same $\eta$ as in (3). We are, accordingly, forced to study the m-point boundary value problem (2) directly and obtain results about the three-point boundary value problem (1) as a corollary to the results for the $\mathrm{m}$-point boundary value probelm. It is interesting to note that while in the nonresonance case we had to study the m-point boundary value problem, using the results for the three -point boundary value problem, it is just the reverse case in the resonance case.

We obtain conditions for the existence of a solution for the boundary value problem (2). using Mawhin's version of the Leray Schauder Continuation theorem [5] or [6] or [7]. Recently, Gupta. Ntouyas and Tsamatos studied the m-point boundary value problem

$$
\begin{gathered}
x^{\prime \prime}(t)=f\left(t, x(t), x^{\prime}(t)\right)+e(t), 0<t<1, \\
x^{\prime}(0)=0, x(1)=\sum_{t=1}^{m-2} a_{1} x\left(\xi_{1}\right),
\end{gathered}
$$

with $\xi_{1} \in(0,1) .0<\xi_{1}<\xi_{2}<\cdots<\xi_{m-2}<1, a_{1} \in R$, all $a_{1}$ having the same sign, given, and $\sum_{t=1}^{m-2} a_{1} \neq 1$, in [3]. The boundary value problem (2) differs from the boundary value problem (4) in that the associated linear boundary value problem with (2), namely,

$$
\begin{gathered}
x^{\prime \prime}(t)=0,0<t<1, \\
x^{\prime}(0)=0, x(1)=\sum_{t=1}^{m-2} a_{1} x\left(\xi_{1}\right),
\end{gathered}
$$

has $x(t)=A$, for $A \in R$, as non-trivial solutions, since $\sum_{t=1}^{m-2} a_{t}=1$, while the corresponding linear boundary value problem associated with (4), namely,

$$
\begin{gathered}
x^{\prime \prime}(t)=0,0<t<1, \\
x^{\prime}(0)=0, x(1)=\sum_{t=1}^{m-2} a_{1} x\left(\xi_{2}\right),
\end{gathered}
$$

with $\sum_{t=1}^{m-2} a_{\imath} \neq 1$, has $x(t) \equiv 0$, as its only solution. It is for this reason we call the boundary value problem (2) to be at resonance. For some recent results on m-point and three-point boundary value problems we refer the reader to [2], [3], [4], [8], [9], [10], (and [11]).

We use the classical spaces $C[0,1], C^{k}[0,1], L^{k}[0,1]$, and $L^{\infty}[0,1]$ of continuous, $k$-times continuously differentiable, measurable real-valued functions whose $k$-th power of the absolute value is Lebesgue integrable on $[0,1]$, or measurable functions that are essentially bounded on $[0,1]$. We also use the Sobolev space $W^{2, k}(0,1), k=1,2$ defined by

$$
W^{2, k}(0,1)=\left\{x:[0,1] \rightarrow R \mid x, x^{\prime} \text { abs. cont. on }[0,1] \text { with } x^{\prime \prime} \in L^{k}[0,1]\right\}
$$

with its usual norm. We denote the norm in $L^{k}[0,1]$ by $\|.\|_{k}$, and the norm in $L^{\infty}[0,1]$ by $\|.\|_{\infty}$.

\section{EXISTENCE THEOREMS.}

Let $X, Y$ denote Banach spaces $X=C^{1}[0,1]$ and $Y=L^{1}[0,1]$ with their usual norms. Let $Y_{2}$ be the subspace of $Y$ spanned by the function 1, i.e.

$$
Y_{2}=\{x(t) \in Y \mid x(t)=A \text {, a.e. on }[0,1], A \in R\}
$$

and let $Y_{1}$ be the subspace of $Y$ such that $Y=Y_{1} \oplus Y_{2}$. Let $a_{1} \geq 0, \xi_{1} \in(0,1), i=1,2, \cdots, m-2$ with $\sum_{i=1}^{m-2} a_{1}=1,0<\xi_{1}<\xi_{2}<\cdots,<\xi_{m-2}<1$, be given. We note that for $x(t) \in Y$ we can write

$$
x(t)=(x(t)-A)+A,
$$

with $A=\frac{2}{\sum_{t=1}^{m-2} a_{1}\left(1-\xi_{1}^{2}\right)} \sum_{t=1}^{m-2} a_{1}\left[\int_{0}^{\xi_{1}}\left(1-\xi_{i}\right) x(s) d s+\int_{\xi_{1}}^{1}(1-s) x(s) d s\right]$, for $t \in[0,1]$. We define the canonical projection operators $P: Y \rightarrow Y_{1}, Q: Y \rightarrow Y_{2}$ by

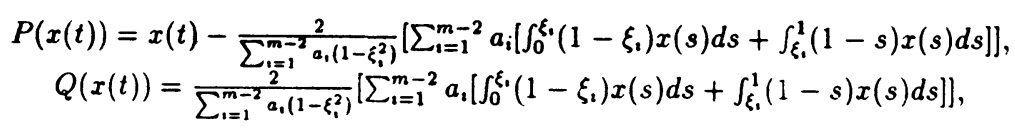

for $x(t) \in Y$. We note that if $Q(x(t))=0$, there exists a $\zeta \in(0,1)$ such that $x(\zeta)=0$. Clearly, $Q=I-P$, where $I$ denotes the identity mapping on $Y$, and the projections $P$ and $Q$ are continuous. Now let $X_{2}=X \cap Y_{2}$. Clearly $X_{2}$ is a closed subspace of $X$. Let $X_{1}$ be the closed subspace of $X$ such 
that $X=X_{1} \oplus X_{2}$. We note that $P(X) \subset X_{1}, Q(X) \subset X_{2}$ and the projections $P \mid X: X \rightarrow X_{1}$ and $Q \mid X: X \rightarrow X_{2}$ are continuous. In the following, $X, Y, P, Q$ will refer to the Banach spaces and the projections as defined and we shall not distinguish between $P, P \mid X$ (resp. $Q, Q \mid X$ ) and depend on the context for the proper meaning.

Define a linear operator $L: D(L) \subset X \rightarrow Y$ by setting

$$
D(L)=\left\{x \in W^{2,1}(0,1) \mid x^{\prime}(0)=0, x(1)=\sum_{i=1}^{m-2} a_{i} x\left(\xi_{i}\right)\right\},
$$

and for $x \in D(L)$,

$$
L x=x^{\prime \prime} .
$$

Let, now, for $e \in Y_{1}$, i.e. $e \in L^{1}[0,1]$ with $\sum_{t=1}^{m-2} a_{t}\left[\int_{0}^{\xi_{1}}\left(1-\xi_{1}\right) e(s) d s+\int_{\xi_{1}}^{1}(1-s) e(s) d s\right]=0, K e$ denote the unique solution of the boundary value problem

$$
\begin{gathered}
x^{\prime \prime}(t)=e(t), 0<t<1, \\
x^{\prime}(0)=0, x(1)=\sum_{t=1}^{m-2} a_{1} x\left(\xi_{\imath}\right),
\end{gathered}
$$

such that $\sum_{t=1}^{m-2} a_{1}\left[\int_{0}^{\xi_{1}}\left(1-\xi_{1}\right) x(s) d s+\int_{\xi_{1}}^{1}(1-s) x(s) d s\right]=0$. Indeed, for $t \in[0,1]$,

$$
(K e)(t)=\int_{0}^{t}(t-s) e(s) d s+A,
$$

where $A=-\frac{2}{\sum_{r=1}^{m-2} a_{i}\left(1-\xi_{1}^{2}\right)}\left[\sum_{t=1}^{m-2} a_{1}\left[\int_{0}^{\xi_{1}} \int_{0}^{t}\left(1-\xi_{i}\right)(t-s) e(s) d s d t+\int_{\xi_{1}}^{1} \int_{0}^{t}(1-t)(t-s) e(s) d s d t\right]\right]$. Accordingly the linear mapping $K: Y_{1} \rightarrow X_{1}$ defined by the equation (12) is a bounded linear mapping and is such that for

$$
x \in Y, K P x \in D(L) \text {, and } L K P(x)=P(x) .
$$

DEFINITION 1 :- A function $f:[0,1] \times R^{2} \rightarrow R$ satisfies Caratheodory's conditions if (i) for each $(x, y) \in R^{2}$, the function $t \in[0,1] \rightarrow f(t, x, y) \in R$ is measurable on $[0,1]$, (ii) for a.e. $t \in[0,1]$, the function $(x, y) \in R^{2} \rightarrow f(t, x, y) \in R$ is continuous on $R^{2}$, and (iii) for each $r>0$, there exists $\alpha_{r}(t) \in L^{1}[0,1]$ such that $|f(t, x, y)| \leq \alpha_{r}(t)$ for a.e. $t \in[0,1]$ and all $(x, y) \in R^{2}$ with $\sqrt{x^{2}+y^{2}} \leq r$.

Let $f:[0,1] \times R^{2} \rightarrow R$ be a function satisfying Caratheodory's conditions. Let $N: X \rightarrow Y$ be the non-linear mapping defined by

$$
(N x)(t)=f\left(t, x(t), x^{\prime}(t)\right), t \in[0,1],
$$

for $x(t) \in X$.

For $e(t) \in Y_{1}$, i.e. $e(t) \in L^{1}[0,1]$ with $\sum_{i=1}^{m-2} a_{i}\left[\int_{0}^{\xi_{1}}\left(1-\xi_{1}\right) e(s) d s+\int_{\xi_{1}}^{1}(1-s) e(s) d s\right]=0$, the boundary value problem $(2)$ reduces to the functional equation

$$
L x=N x+e,
$$

in $X$, with $e(t) \in Y_{1}$, given.

THEOREM 2 :- Let $f:[0,1] \times R^{2} \rightarrow R$ be a function satisfying Caratheodory's conditions. Assume that there exist functions $p(t), q(t), r(t)$ in $L^{1}(0,1)$ such that

$$
\left|f\left(t, x_{1}, x_{2}\right)\right| \leq p(t)\left|x_{1}\right|+q(t)\left|x_{2}\right|+r(t)
$$

for a.e. $t \in[0,1]$ and all $\left(x_{1}, x_{2}\right) \in R^{2}$. Also let $a_{i} \geq 0, \xi_{i} \in(0,1), i=1,2, \cdots, m-2$ with $\sum_{i=1}^{m-2} a_{i}=1,0<\xi_{1}<\xi_{2}<\cdots,<\xi_{m-2}<1$ be given, and assume that for every $x(t) \in X$,

$$
(Q x)(t) \cdot(Q N x)(t) \geq 0, \text { for } t \in[0,1] .
$$

Then for $e(t) \in Y_{1}$, i.e. $e(t) \in L^{1}[0,1]$ with $\sum_{t=1}^{m-3} a_{1}\left[\int_{0}^{\xi_{1}}\left(1-\xi_{1}\right) e(s) d s+\int_{\xi_{1}}^{1}(1-s) e(s) d s\right]=0$, given, the boundary value problem (2) has at least one solution in $C^{1}[0,1]$ provided

$$
\|p\|_{1}+\|q\|_{1}<1
$$


PROOF:- We first note that the bounded linear mapping $K: Y_{1} \rightarrow X_{1}$ defined by the equation (12) is such that the mapping $K P N: X \rightarrow X$ maps bounded subsets of $X$ into relatively compact subsets of $X$, in view of Arzela-Ascoli Theorem. Hence $K^{\prime} P N: X \rightarrow X$ is a compact mapping.

We, next, note that $x \in C^{1}[0,1]$ is a solution of the boundary value problem (2) if and only if $x$ is a solution to the operator equation

$$
L x=N x+e .
$$

Now, to solve the operator equation $L x=N x+e$, it suffices to solve the system of equations

$$
\begin{gathered}
P x=K P N x+e_{1}, \\
Q N x=0,
\end{gathered}
$$

$x \in X, e_{1}=K e$ (note that since $e \in Y_{1}, P e=e, Q e=0$ ). Indeed, if $x \in X$ is a solution of (17) then $x \in D(L)$ and

$$
\begin{gathered}
L P x=L x=L K P N x+L e_{1}=P N x+e, \\
Q N x=0,
\end{gathered}
$$

which gives on adding that $L x=N x+e$.

Now, (17) is clearly equivalent to the single equation

$$
P x+Q N x-K P N x=e_{1},
$$

which has the form of a compact perturbation of the Fredholm operator $P$ of index zero. We can, therefore, apply the version given in ([5], Theorem 1, Corollary 1) or ([6], Theorem IV.4) or ([7]) of the Leray-Schauder Continuation theorem which ensures the existence of a solution for (18) if the set of all possible solutions of the family of equations

$$
P x+(1-\lambda) Q x+\lambda Q N x-\lambda K P N x=\lambda e_{1},
$$

$\lambda \in(0,1)$, is a priori bounded, independently of $\lambda$. Notice that (19) is then equivalent to the system of equations

$$
\begin{gathered}
P x=\lambda K P N x+\lambda e_{1}, \\
(1-\lambda) Q x+\lambda Q N x=0 .
\end{gathered}
$$

Let, now, $x(t)$ be a solution of $(20)$ for some $\lambda \in(0,1)$. We see on multiplying the second equation in (20) and using (15) that $(1-\lambda)((Q x)(t))^{2} \leq 0$ for every $t \in[0,1]$. Hence $(Q x)(t)=0$ for every $t \in[0,1]$ and accordingly there exists a $\zeta \in(0,1)$ such that $x(\zeta)=0$. Since, now, $x^{\prime}(0)=0$ it follows that $\|x\|_{\infty} \leq\left\|x^{\prime}\right\|_{\infty} \leq\left\|x^{\prime \prime}\right\|_{1}$. Also since $Q x=0$, we have $Q N x=0$. It follows that $x \in D(L)$, i.e., $x \in W^{2,1}(0,1)$ with $x^{\prime}(0)=0, x(1)=\sum_{s=1}^{m-2} a_{1} x\left(\xi_{t}\right)$ and $x^{\prime \prime}(t)=\lambda f\left(t, x(t), x^{\prime}(t)\right)+\lambda e(t)$. Accordingly, we get that

$$
\begin{gathered}
\left\|x^{\prime \prime}\right\|_{1}=\lambda\left\|f\left(t, x(t), x^{\prime}(t)\right)+e(t)\right\|_{1} \\
\leq\|p\|_{1}\|x\|_{\infty}+\|q\|_{1}\left\|x^{\prime}\right\|_{\infty}+\|r\|_{1}+\|e\|_{1} \\
\leq\left(\|p\|_{1}+\|q\|_{1}\right)\left\|x^{\prime \prime}\right\|_{1}+\|r\|_{1}+\|e\|_{1}
\end{gathered}
$$

It follows from the assumption (16) that there is a constant $c$, independent of $\lambda \in(0,1)$ and $x(t)$, such that

$$
\left\|x^{\prime \prime}\right\|_{1} \leq c \text {. }
$$

It is now immediate from $\|x\|_{\infty} \leq\left\|x^{\prime}\right\|_{\infty} \leq\left\|x^{\prime \prime}\right\|_{1}$ that the set of solutions of the family of equations (20) is, a priori, bounded in $C^{1}[0,1]$ by a constant, independent of $\lambda \in(0,1)$.

This completes the proof of the theorem.//

REMARK 1:- We remark that the Theorem 2 remains valid if we replace (15) by the condition

$$
(Q x)(t) \cdot(Q N x)(t) \leq 0, \text { for } t \in[0,1]
$$

for every $x \in X$.

REMARK 2:- We remark that the condition (15) can be replaced by the condition

$$
f\left(t, x_{1}, x_{2}\right) x_{1} \geq 0
$$


for almost all $t \in(0,1)$ and all $\left(x_{1}, x_{2}\right) \in R^{2}$. Indeed, condition (15) was used to show, in the proof of Theorem 2, that if $x(t)$ is a solution of $(20)$ for some $\lambda \in(0,1)$ then there exists a $\zeta \in(0,1)$ such that $x(\zeta)=0$. We, now, show that (22), implies that if $x(t)$ is a solution of $(20)$ for some $\lambda \in(0,1)$ then there exists a $\zeta \in(0,1)$ such that $x(\zeta)=0$. Indeed, suppose that $x(t) \neq 0$, for all $t \in(0,1)$. We may, infact, assume without any loss of generality that $x(t)>0$, for every $t \in(0,1)$. It then follows from (22) that $f\left(t . x(t), x^{\prime}(t)\right) \geq 0$. for a.e. $t \in(0.1)$. Hence $Q x>0$ and $Q N x \geq 0$. Now the second equation in $(20)$ gives that $(1-\lambda)(Q x)^{2}+\lambda(Q N x)(Q x)=0$, so that we get $(Q x)^{2} \leq 0$, a contradiction. Accordingly, there must exist a $\zeta \in(0,1)$ such that $x(\zeta)=0$.

THEOREM 3 :- Let $f:[0,1] \times R^{2} \rightarrow R$ be a function as in Theorem 2. Assume that the functions $p(t), q(t), r(t)$ in (14) are in $L^{2}(0,1)$. Let $a_{\imath} \geq 0, \xi_{\imath} \in(0,1), \imath=1,2, \cdots, m-2$ with $\sum_{t=1}^{m-2} a_{\mathbf{t}}=1$, $0<\xi_{1}<\xi_{2}<\ldots,<\xi_{m-2}<1$ be given.

Then for $e(t) \in L^{2}[0,1]$ with $\sum_{t=1}^{m-2} a_{1} \int_{\xi_{1}}^{1} e(s) d s=0$, given, the boundary value problem (2) has at least one solution in $C^{1}[0,1]$ provided

$$
\frac{2}{\pi}\left(\frac{2}{\pi}\|p\|_{2}+\|q\|_{2}\right)<1 .
$$

PROOF:- The proof is similar to the proof of Theorem 2, except now one uses the inequalities $\|x\|_{2} \leq \frac{2}{\pi}\left\|x^{\prime}\right\|_{2} \leq \frac{4}{\pi^{2}}\left\|x^{\prime \prime}\right\|_{2}$ for an $x \in W^{2} 2(0,1)$ with $x(\zeta)=0$, for some $\zeta \in(0,1)$ and $x^{\prime}(0)=0$ (see, Theorem 256 of [12]) to show that the set of solutions of the family of equations (19) is a priori bounded in $C^{1}[0,1]$ by a constant independent of $\lambda \in(0,1) . / /$

THEOREM 4 :- Let $f:[0,1] \times R^{2} \rightarrow R$ be a function as in Theorem 2 (respectively, Theorem 3). Let $\eta \in(0,1)$ be given. Then for $\epsilon(t) \in L^{1}[0,1]$ (resectively. $\left.e(t) \in L^{2}[0,1]\right)$ with $\int_{0}^{\eta}(1-\eta) e(s) d s+$ $\int_{\eta}^{1}(1-s) e(s) d s=0$, given, the thret-point boundary value problem (1) has at least one solution in $C^{1}[0,1]$ provided

$$
\|p\|_{1}+\|q\|_{1}<1
$$

(respectively, $\left.\frac{2}{\pi}\left(\frac{2}{\pi}\|p\|_{2}+\|q\|_{2}\right)<1\right)$.

PROOF:- The theorem follows immediately from Theorem 2 (respectively, Theorem 3) with $m=3$ and $a_{1}=1, \xi_{1}=\eta . / /$

THEOREM 5 :- Let $f:[0,1] \times R^{2} \rightarrow R$ be a function as in Theorem 2 (respectively, Theorem 3). Then for $e(t) \in L^{1}[0,1]$ (resectively, $e(t) \in L^{2}[0,1]$ ) with $\int_{0}^{1}(1-s) e(s) d s=0$, given, the boundary value problem

$$
\begin{gathered}
x^{\prime \prime}(t)=f\left(t, x(t), x^{\prime}(t)\right)+e(t), 0<t<1, \\
x^{\prime}(0)=0, x(0)=x(1),
\end{gathered}
$$

has at least one solution in $C^{1}[0,1]$ provided

$$
\|p\|_{1}+\|q\|_{1}<1
$$

(respectively, $\left.\frac{2}{\pi}\left(\frac{2}{\pi}\|p\|_{2}+\|q\|_{2}\right)<1\right)$.

PROOF:- The theorem follows immediately from Theorem 2 (respectively, Theorem 3) with $m=2$ and $a_{1}=1, \xi_{1}=0 . / /$

\section{References}

[1] V. A. Il'in, and E. I. Moiseev, Nonlocal boundary value problem of the first kind for a SturmLiouville operator in its differential and finite difference aspects, Differential Equations. Vol. 23, No.27, (1988) pp. 803-810.

[2] C. P. Gupta, S. K. Ntouyas, and P. Ch. Tsamatos, On an m-point boundary value problem for second order ordinary differential equations. International Journal of Nonlinear Analysis, TMAA (to appear). 
[3] C. P. Gupta, S. K. Ntouyas, and P. Ch. Tsamatos, Solvability of an m-point boundary ralue problem for second order ordinary differential equations. Jour. Math. Anal. Appl., (to appear)

[4] C. P. Gupta, S. K. Ntoiuyas, and P. Ch. Tsamatos, Existence results for m-point boundary value problems. Journal of Differential equations and Dynamical Systems (to appear)

[5] J. Mawhin, Landesman-Lazer type problems for nonlinear equations, Conference Sem. Mat. Univ. Bari, 147 (1977)

[6] J. Mawhin, Topological Degree methods in Nonlinear Boundary Value Problems, in "NSF. CBMS Regional Conference Series in Math." No. 40, Amer. Math. Soc., Providence, RI, 1979

[7] J. Mawhin, Compacité, Monotonie et Convexité dans letude de Problèmes aux Limite Semilinéaires, Sem. Anal. Moderne 19 (1981)

[8] C. P. Gupta, Solvability of a three-point boundary value problem for a second order ordinary differential equation, Jour. Math. Anal. Appl., Vol. 168 (1992), pp. 540-551

[9] C. P. Gupta, A note on a second order three-point boundary value problem, Jour. Math. Anal. Appl., (to appear).

[10] C. P. Gupta, Solvability of a multi-point boundary value problem at resonance. Results in Mathematics (to appear)

[11] S. A. Marano, A remark on a second order three-point boundary value problem, Jour. Math. Anal. Appl., (to appear)

[12] G. H. Hardy, J. E. Littlewood, and G. Polya, Incqualities, Cambridge University Press, London/New York, 1967. 


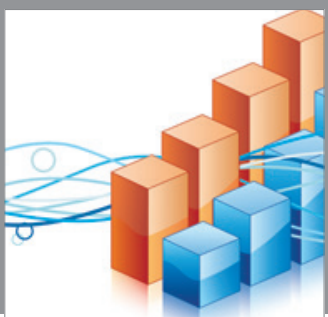

Advances in

Operations Research

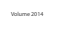

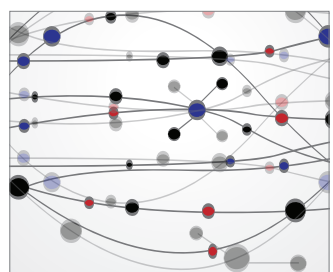

\section{The Scientific} World Journal
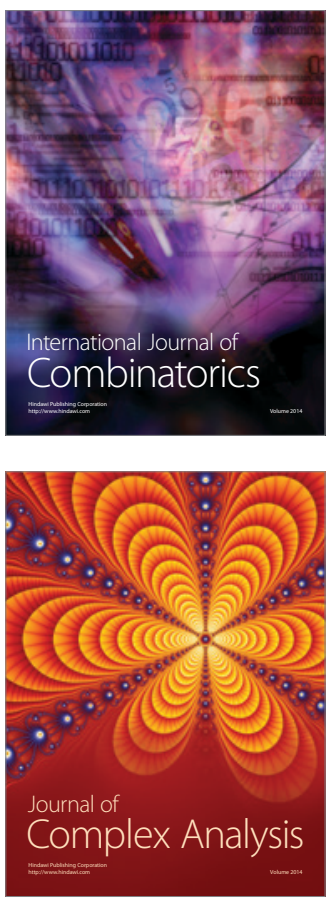

International Journal of

Mathematics and

Mathematical

Sciences
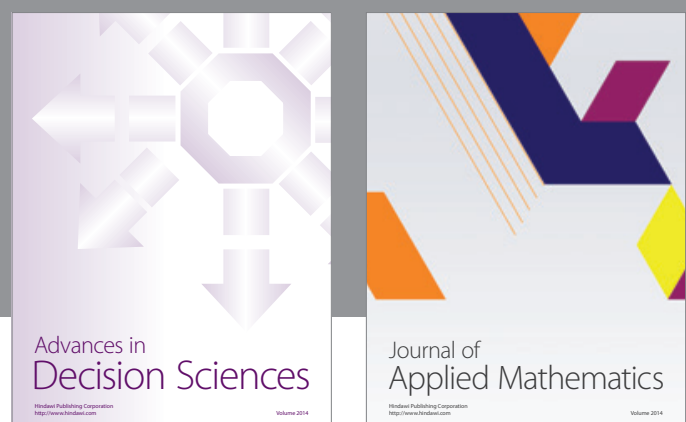

Journal of

Applied Mathematics
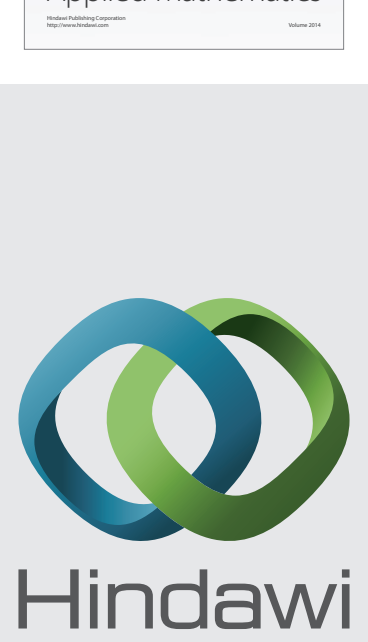

Submit your manuscripts at http://www.hindawi.com
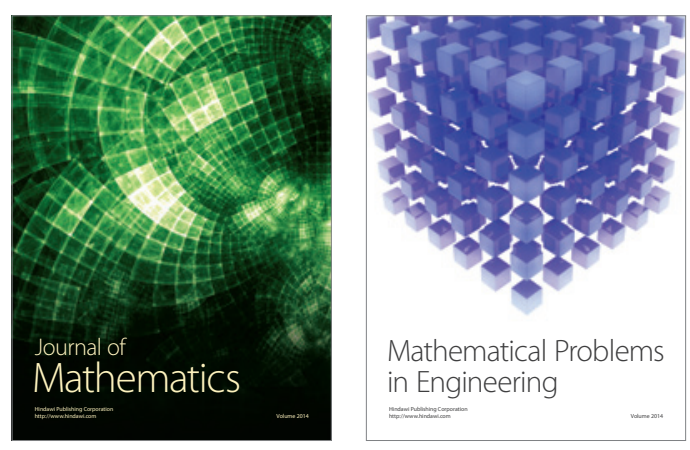

Mathematical Problems in Engineering
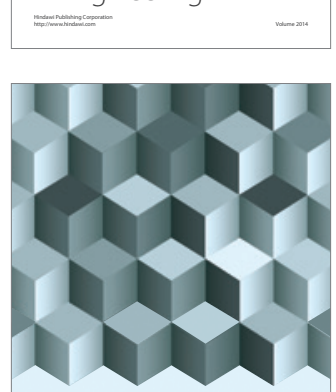

Journal of

Function Spaces
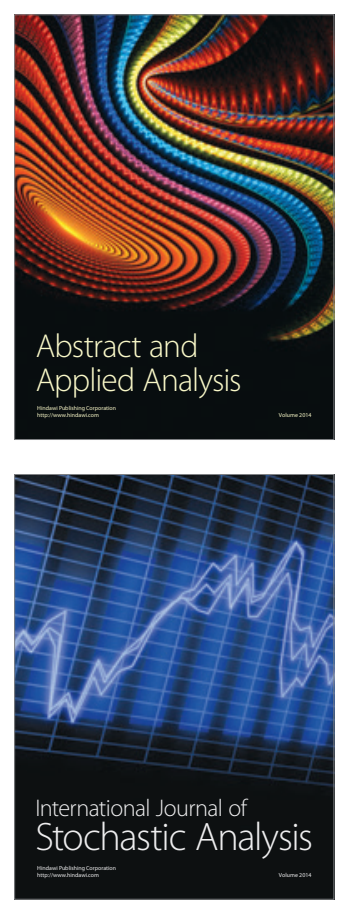

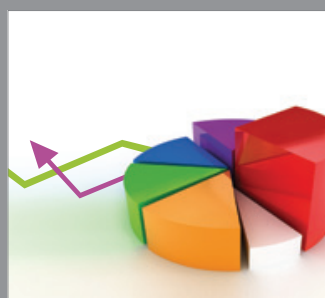

ournal of

Probability and Statistics

Promensencen
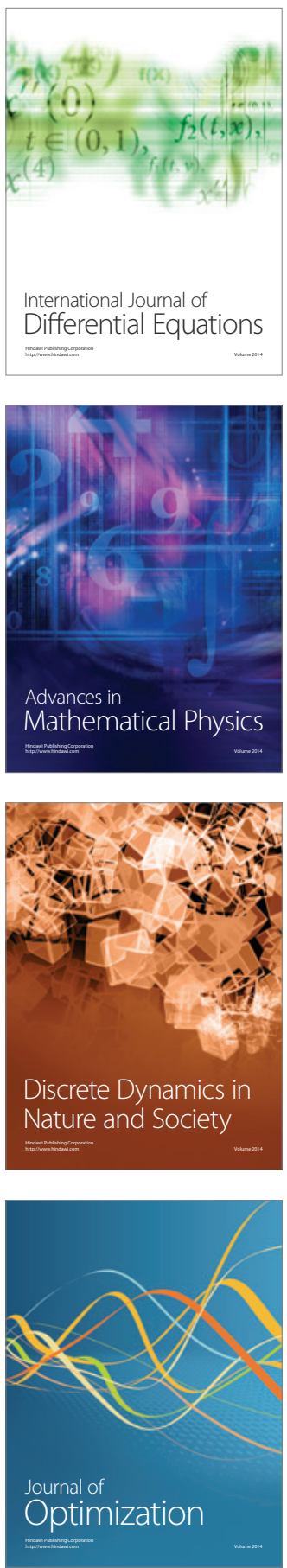